\title{
Erratum to: Genome-wide analysis of the regulation of pimaricin production in Streptomyces natalensis by reactive oxygen species
}

\author{
Tiago Beites • Antonio Rodríguez-García • Fernando Santos-Beneit • \\ Pedro Moradas-Ferreira • Jesús F. Aparicio • Marta V. Mendes
}

Published online: 7 February 2014

(C) Springer-Verlag Berlin Heidelberg 2014

\section{Erratum to: Appl Microbiol Biotechnol}

DOI 10.1007/s00253-013-5455-Z

The original version of this article inadvertently contained mistake. The author "Fernando Santos-Beneit" was omitted from the author list.

The correct list of author's is presented above.

The online version of the original article can be found at http://dx.doi.org/ 10.1007/s00253-013-5455-z.

T. Beites $\cdot$ P. Moradas-Ferreira $\cdot$ M. V. Mendes $(\square)$

Instituto de Biologia Molecular e Celular (IBMC), Universidade do

Porto, Rua do Campo Alegre 823, 4150-180 Porto, Portugal

e-mail: mvm@ibmc.up.pt

T. Beites

Departamento de Biologia, FCUP-Faculdade de Ciências,

Universidade do Porto, Porto, Portugal. Rua do Campo Alegre,

Edifício FC4, 4169-007 Porto, Portugal

A. Rodríguez-García $\cdot$ F. Santos-Beneit • J. F. Aparicio Instituto de Biotecnología de León (INBIOTEC), Avenida Real 1,

24006 León, Spain

A. Rodríguez-García $\cdot$ F. Santos-Beneit • J. F. Aparicio

Área de Microbiología, Facultad de Biología, Universidad de León,

Campus de Vegazana s/n, 24071 León, Spain

P. Moradas-Ferreira

Instituto de Ciências Biomédicas Abel Salazar (ICBAS),

Universidade do Porto, Rua Jorge Viterbo Ferreira 228,

4050-313 Porto, Portugal 